Archived version from NCDOCKS Institutional Repository http://libres.uncg.edu/ir/asu/

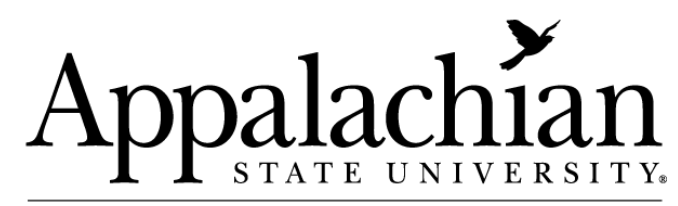

B O O N E, NORT H C A R O L I A

\title{
Effect Of N-3 Fatty Acids And Antioxidants On Oxidative Stress After Exercise
}

\author{
BY: STEVEN R. MCANULTY, DAVID C. NIEMAN, MASHA FOX-RABINOVICH, VALERIE DURAN, \\ LISA S. MCANULTY, DRU A. HENSON, FUXIA JIN, and MICHAEL J. LANDRAM
}

\begin{abstract}
Purpose: $\mathrm{n}-3$ fatty acids are known to exert multiple beneficial effects including anti-inflammatory actions that may diminish oxidative stress. Supplementation with antioxidant vitamins has been proposed to counteract oxidative stress and improve antioxidant status. Therefore, this project investigated the effects of daily supplementation in 48 trained cyclists over $6 \mathrm{wk}$ and during $3 \mathrm{~d}$ of continuous exercise on F2-isoprostanes (oxidative stress), plasma n-3 fatty acids, and antioxidant status (oxygen radical absorption capacity and ferricreducing antioxidant potential). Methods: Cyclists were randomized into $n-3$ fatty acids (N3) $(n=11)(2000$ $\mathrm{mg}$ of eicosapentaenoic acid and $400 \mathrm{mg}$ of docosahexaenoic acid), a vitamin-mineral (VM) complex ( $\mathrm{n}=12)$ emphasizing vitamins $C(2000 \mathrm{mg}), E(800 \mathrm{IU}), \mathrm{A}(3000 \mathrm{IU})$, and selenium $(200 \mathrm{Kg})$, a VM and n-3 fatty acid combination (VN3) ( $n=13)$, or placebo $(P)(n=12)$. Blood was collected at baseline and preexercise and postexercise. A $4 \times 3$ repeated-measures ANOVA was performed to test main effects. Results: After exercise, F2isoprostanes were higher in N3 (treatment effect $P=0.014$ ). Eicosapentaenoic acid and docosahexaenoic acid plasma values were higher after supplementation (interaction effect $P=0.001$ and 0.006 , respectively) in both n-3 supplemented groups. Oxygen radical absorption capacity declined similarly among all groups after exercise. Ferric-reducing antioxidant potential exhibited significant interaction $(P=0.045)$ and significantly increased after exercise in VN3 and VM (P G 0.01). Conclusions: This study indicates that supplementation with n-3 fatty acids alone significantly increases F2-isoprostanes after exhaustive exercise. Lastly, antioxidant supplementation augments plasma antioxidant status and modestly attenuates but does not prevent the significant n-3 fatty acid associated increase in F2-isoprostanes postexercise.
\end{abstract}

MCANULTY, STEVEN R. 1 ; NIEMAN, DAVID C. 1 ; FOX-RABINOVICH, MASHA2; DURAN, VALERIE2; MCANULTY, LISA S.2; HENSON, DRU A.3; JIN, FUXIA4; LANDRAM, MICHAEL J.1 (2010). Effect of n-3 Fatty Acids and Antioxidants on Oxidative Stress after Exercise. Medicine \& Science in Sports \& Exercise, Sept. 2010, 42(9): 1704-1711. doi: 10.1249/MSS.0b013e3181 d85bd1. Publisher version of record available at: https:// journals.Iww.com/acsm-msse/Fulltext/2010/09000/Effect_of_n_3_Fatty_Acids_and_Antioxidants_on.13.aspx 


\title{
Effect of n-3 Fatty Acids and Antioxidants on Oxidative Stress after Exercise
}

\author{
STEVEN R. MCANULTY ${ }^{1}$, DAVID C. NIEMAN ${ }^{1}$, MASHA FOX-RABINOVICH ${ }^{2}$, VALERIE DURAN ${ }^{2}$, \\ LISA S. MCANULTY ${ }^{2}$, DRU A. HENSON ${ }^{3}$, FUXIA JIN ${ }^{4}$, and MICHAEL J. LANDRAM ${ }^{1}$
}

${ }^{I}$ Department of Health, Leisure, and Exercise Science, Appalachian State University, Boone, $N C$; ${ }^{2}$ Department of Family and Consumer Science, Appalachian State University, Boone, $N C ;{ }^{3}$ Department of Biology, Appalachian State University, Boone, NC; and ${ }^{4}$ Chemistry, Appalachian State University, Boone, $N C$

\begin{abstract}
McANUlTY, S. R., D. C. NIEMAN, M. FOX-RABINOVICH, V. DURAN, L. S. MCANULTY, D. A. HENSON, F. JIN, and M. J. LANDRAM. Effect of n-3 Fatty Acids and Antioxidants on Oxidative Stress after Exercise. Med. Sci. Sports Exerc., Vol. 42, No. 9, pp. 1704-1711, 2010. Purpose: n-3 fatty acids are known to exert multiple beneficial effects including anti-inflammatory actions that may diminish oxidative stress. Supplementation with antioxidant vitamins has been proposed to counteract oxidative stress and improve antioxidant status. Therefore, this project investigated the effects of daily supplementation in 48 trained cyclists over $6 \mathrm{wk}$ and during $3 \mathrm{~d}$ of continuous exercise on $\mathrm{F}_{2}$-isoprostanes (oxidative stress), plasma $\mathrm{n}-3$ fatty acids, and antioxidant status (oxygen radical absorption capacity and ferric-reducing antioxidant potential). Methods: Cyclists were randomized into n-3 fatty acids (N3) ( $n=11$ ) (2000 mg of eicosapentaenoic acid and $400 \mathrm{mg}$ of docosahexaenoic acid), a vitamin-mineral (VM) complex $(n=12)$ emphasizing vitamins C (2000 mg), E (800 IU), A (3000 IU), and selenium (200 $\mu \mathrm{g})$, a VM and n-3 fatty acid combination (VN3) $(n=13)$, or placebo (P) $(n=12)$. Blood was collected at baseline and preexercise and postexercise. A $4 \times 3$ repeated-measures ANOVA was performed to test main effects. Results: After exercise, $\mathrm{F}_{2}$-isoprostanes were higher in N3 (treatment effect $P=0.014$ ). Eicosapentaenoic acid and docosahexaenoic acid plasma values were higher after supplementation (interaction effect $P=0.001$ and 0.006 , respectively) in both $\mathrm{n}-3$ supplemented groups. Oxygen radical absorption capacity declined similarly among all groups after exercise. Ferric-reducing antioxidant potential exhibited significant interaction $(P=0.045)$ and significantly increased after exercise in VN3 and VM $(P<0.01)$. Conclusions: This study indicates that supplementation with $\mathrm{n}-3$ fatty acids alone significantly increases $\mathrm{F}_{2}$-isoprostanes after exhaustive exercise. Lastly, antioxidant supplementation augments plasma antioxidant status and modestly attenuates but does not prevent the significant $n-3$ fatty acid associated increase in $\mathrm{F}_{2}$-isoprostanes postexercise. Key Words: EICOSAPENTAENOIC ACID, DOCOSAHEXAENOIC ACID, F2-ISOPROSTANES, CYCLING
\end{abstract}

$\mathrm{O}$ mega-3 fatty acids (n-3fa) comprise a family of unsaturated fatty acids that consist of $\alpha$-linolenic acid, eicosapentaenoic acid (EPA), and docosahexaenoic acid (DHA), which are essential nutrients that must be obtained from food because of the inability of humans to synthesize these fatty acids de novo (28). Human beings evolved consuming a diet that contained about equal amounts of omega- 6 (n-6fa) and n-3fa fatty acids. Current western diets provide ratios of $n-6 \mathrm{fa}$ to $\mathrm{n}-3 \mathrm{fa}$ ranging from approximately $10: 1$ to $20: 1$ instead of the traditional range of $1: 1$ to $2: 1$, respectively. It is known that a high intake of $\mathrm{n}-6 \mathrm{fa}$ increases prothrombotic and proaggregatory activities

Address for correspondence: Steven R. McAnulty, Ph.D., Dept HLES, PO Box 32071, Appalachian State University, Boone, NC 28608; Email: mcanltysr@appstate.edu. characterized by increases in blood viscosity and vasospasm and vasoconstriction (27). Given that $n-3$ fa exhibit antiinflammatory, antithrombotic, antiarrhythmic, hypolipidemic, and vasodilatory properties, an increased consumption of n-3fa would likely be beneficial (27).

Although $n-3 f a$ have potential benefits against certain diseases such as cardiovascular disease, Alzheimer's disease, Parkinson's disease, diabetes mellitus, essential hypertension, and atherosclerosis, questions remain about the effect on subsequent oxidative stress if supplemented alone or in conjunction with other antioxidants $(4,8,15,26)$. Specifically, increased consumption of n-3fa may lead to increased lipid peroxidation (18). Thus far, the data obtained in vivo are not conclusive for (18) or against (1) increased lipid peroxidation with n-3 fatty acid supplementation. Furthermore, a study that has examined n-3fa supplementation and oxidative damage with exercise has not been conducted to our knowledge. Therefore, it is not known whether heavily exercising athletes should supplement with n-3fa.

It is well established that a causal link exits between oxidative stress and various disease states such as cancer and heart disease $(2,7)$. Routine exercise is generally regarded as 
being beneficial and helpful in disease and mortality prevention (5). Somewhat paradoxical is the fact that acute exercise results in an increase in oxidative damage (17), but chronic exercise is known to be anti-inflammatory and reduces the amount of damage that occurs during acute exercise (12). Because an excess of oxidative stress is associated with negative consequences, supplementation with antioxidant vitamins has been proposed to counteract oxidative stress and to improve antioxidant status, but the existing literature is equivocal regarding exercise and antioxidant vitamins $(16,17)$. Interestingly, Ristow et al. (25) found that supplementation with antioxidant vitamins actually ameliorated beneficial adaptations from exercise in preventing insulin resistance. Previous antioxidant research by our team indicates that individual antioxidants, even in high doses, are not effective at preventing peroxidation $(17,23)$, but utilization of antioxidant combinations has shown more promise (16).

Because to our knowledge no study has yet been published that directly examines n-3 fatty acid supplementation and direct markers of oxidative damage after exhaustive exercise, the primary purpose of this project was to investigate whether supplementation would increase or decrease $\mathrm{F}_{2}$-isoprostanes. $\mathrm{F}_{2}$-isoprostanes are bioactive compounds that are considered to be the gold standard for determination of oxidative stress. Interestingly, $\mathrm{F}_{2}$-isoprostanes are elevated in obesity and various other disease states associated with states of elevated oxidative stress (20). Second, we wished to examine whether antioxidant vitamins might directly attenuate $\mathrm{F}_{2}$-isoprostanes when used as the only supplement or reduce potential increases in $\mathrm{F}_{2}$-isoprostanes associated with n-3fa peroxidation. We hypothesized that $\mathrm{n}-3$ fa and antioxidant vitamins would reduce oxidative stress and enhance antioxidant status.

\section{METHODS}

Subjects. Forty-eight trained cyclists were recruited and randomized into vitamin-mineral $(n=12)(\mathrm{VM}), \mathrm{n}-3 \mathrm{fa}$ $(n=11)(\mathrm{N} 3)$, vitamin and $\mathrm{n}-3 \mathrm{fa}$ combination $(n=13)$ (VN3), or placebo $(n=12)(\mathrm{P})$ groups. Written informed consent was obtained from each subject, and the experimental procedures were approved by the institutional review board of Appalachian State University.

Research design. Before the start of supplementation, subjects reported to the University Human Performance Laboratory for baseline orientation, blood sampling, and measurement of cardiorespiratory fitness. $\dot{\mathrm{V}} \mathrm{O}_{2 \max }$ was determined using a graded maximal protocol $(25 \mathrm{~W}$ increase every $2 \mathrm{~min}$ starting at $150 \mathrm{~W}$ ), with the subjects using their own bicycles on CompuTrainer ${ }^{\mathrm{TM}}$ Pro Model 8001 trainers (RacerMate, Seattle, WA). Oxygen uptake and ventilation were measured using the MedGraphics CPX metabolic system (MedGraphics Corporation, St. Paul, MN). HR was measured using a chest HR monitor (Polar Electro Inc., Woodbury, NY). Basic demographic and training data were obtained through a questionnaire. Body composition was assessed by hydrostatic weighing using an electronic load cell system (Exertech, Dresbach, MN), estimated residual volume, and the Siri equation.

Subjects agreed to avoid the use of large-dose VM supplements (above $100 \%$ of recommended dietary allowances), herbs, and medications known to affect oxidative stress during the entire study. During orientation, a dietitian instructed the subjects to follow a diet moderate in carbohydrate (using a food list) during the weekend before and $3 \mathrm{~d}$ of intensified exercise period. At 12:00-12:30 p.m. before each 3-h cycling bout, subjects ingested a standardized liquid meal (Boost Plus; Mead Johnson Nutritionals, Evansville, IN) at an energy level of $63 \mathrm{~kJ} \cdot \mathrm{kg}^{-1}\left(15 \mathrm{kcal} \cdot \mathrm{kg}^{-1}\right)$. Boost Plus is a nutritionally complete, high-energy oral supplement with an energy density of $6.4 \mathrm{~kJ} \cdot \mathrm{mL}^{-1}\left(1.52 \mathrm{kcal} \cdot \mathrm{mL}^{-1}\right)$ and $16 \%$ of energy as protein, $34 \%$ as fat, and $50 \%$ as carbohydrate. No other food and beverage (other than water) was consumed from this meal until the end of the cycling bout. Subjects ingested $0.5-1 \mathrm{~L}$ of water per hour of cycling.

Supplementation. Subjects ingested the supplements daily for $6 \mathrm{wk}$. Under double-blind procedures, subjects assigned to placebo and n-3fa ingested four soft gel capsules per day (two in the morning on an empty stomach at 7:00-8:00 a.m. and two at 6:00-8:00 p.m. before the evening meal) for $6 \mathrm{wk}$ before and during a 3-d period of intensified exercise. The n-3fa capsules contained a highpotency fish oil concentrate from anchovy and sardines, with soybean oil, natural flavors, tocopherols, canola oil, and citric acid, and provided $2000 \mathrm{mg}$ of EPA and $400 \mathrm{mg}$ of DHA (in four soft gels). The placebo capsules were identical in appearance and contained the same ingredients but without the fish oil concentrate. The multivitaminmineral portion of the supplement contained a mixture of vitamins and minerals with an emphasis on antioxidants including vitamins $\mathrm{C}$, E, and A. Subjects assigned to vitamin and minerals ingested eight tablets per day (four in the morning on an empty stomach at 7:00-8:00 a.m. and four tablets at 6:00-8:00 p.m. before the evening meal) for $6 \mathrm{wk}$ before and during a 3-d period of intensified exercise. A total of eight tablets provided vitamins C (2000 mg), E (800 IU), and A (3000 IU) and selenium (200 $\mu \mathrm{g})$. Subjects on combined n-3fa and vitamin and minerals took a combination of two n-3fa softgels and four vitamin and mineral tablets twice daily as described. To ensure compliance, subjects were called weekly and e-mailed daily for reminders and required to return the empty supplement boxes.

Blood and exercise. Baseline blood samples (blood 1) were collected between 7:30 and 9:00 a.m. after an overnight fast and before supplement ingestion. Before exercise and after 6 wk of supplementation, subjects provided blood samples at 8:00 a.m. after an overnight fast and having avoided exercise training for at least $12 \mathrm{~h}$ and before having ingested the morning dose of supplements (blood 2). Subjects then came back to the laboratory for three consecutive 
days and cycled from 3:00 to 6:00 p.m. at approximately $57 \%$ maximal watts while continuing the daily supplement. During the test sessions, experimental subjects cycled using their own bicycles on CompuTrainer ${ }^{\mathrm{TM}}$ Pro Model 8001 trainers with the exercise load set at approximately $57 \%$ maximal watts. Metabolic measurements were made every 30 min of cycling using the MedGraphics CPX metabolic system to verify workload. Blood samples were obtained within 15 min after completing the third 3 -h exercise bout (6:00 p.m.) (blood 3).

Blood sampling conditions and disposition. Blood samples were drawn from an antecubital vein with subjects in the supine position. A local clinical hematology laboratory used a Coulter STKS instrument (Coulter Electronics, Hialeah, FL) and provided hemoglobin and hematocrit for determination of plasma volume change using the method of Dill and Costill (9). Other blood samples were centrifuged in sodium heparin or EDTA tubes, and plasma was aliquoted and then stored at $-80^{\circ} \mathrm{C}$ before analysis for plasma EPA and DHA, $\mathrm{F}_{2}$-isoprostanes, and plasma antioxidant parameters (ferric-reducing antioxidant potential (FRAP) and oxygen radical absorption capacity (ORAC)).

$\mathbf{F}_{2}$-isoprostanes. Plasma $\mathrm{F}_{2}$-isoprostanes were determined using gas chromatography mass spectrometry (GC-MS) as previously described (21). Briefly, free $\mathrm{F}_{2-}$ isoprostanes were extracted from plasma added to a deuterated [2H4] PGF2 internal standard. The mixture was then added to a C18 Sep Pak column, followed by silica solid phase extractions. $\mathrm{F}_{2}$-isoprostanes were converted to pentafluorobenzyl esters, subjected to thin layer chromatography, and converted to trimethylsilyl ether derivatives. Samples were analyzed by a negative ion chemical ionization GC-MS using an Agilent 6890N gas chromatography interfaced to an Agilent 5975B inert MSD mass spectrometer (Agilent Technologies, Inc. Santa Clara, CA). Select ion monitoring techniques were used to analyze the derivatives of $\mathrm{F}_{2}$-isoprostanes and the internal standard. The height of the two ions monitored $\left(\mathrm{m} / \mathrm{z} 569\right.$ for $\mathrm{F}_{2}$-isoprostanes and $\mathrm{m} / \mathrm{z} 573$ for the internal standard, respectively) were compared to give a concentration of $\mathrm{F}_{2}$-isoprostane in plasma in nanograms or picograms per milliliter.

Plasma EPA and DHA. After addition of $500 \mu \mathrm{g}$ of butylated hydroxytoluene and $20 \mu \mathrm{g}$ of heptadecanoic acid methyl ester (NU-Chek Prep, Inc. Elysian, MN) internal standard to $100 \mu \mathrm{L}$ thawed plasma, lipids were extracted, and methyl esters were formed after the mixture was added to $2 \mathrm{~mL}$ of methanolic $5 \% \mathrm{HCl}$ and incubated at $80^{\circ} \mathrm{C}$ for $2 \mathrm{~h}$ in an OLS200 Shaking Waterbath (Grant Instruments Ltd., Shepreth, Cambridgeshire, SG8 6 GB England). The samples were cooled to room temperature upon completion of the incubation, and the methyl esters were extracted twice with $2 \mathrm{~mL}$ of $\mathrm{n}$-hexane. The top layer of the supernatant was combined and dried with N-EVAP116 Nitrogen Evaporator (Organomation Associates, Inc. Berlin, MA). The dried extract was then reconstituted in $200 \mu \mathrm{L}$ of n-hexane supplemented with $0.05 \%$ butylated hydroxytoluene, of which $1 \mu \mathrm{L}$ was injected into an HP $6890 \mathrm{~N}$ gas chromatograph (Agilent Technologies, Palo Alto, CA) equipped with a 5975B Inert XL MSD mass spectrometer detector. A DB-WAX GC column $(30 \mathrm{~m} \times 320 \mu \mathrm{m} \times$ $0.25 \mu \mathrm{m}$ ) from $\mathrm{J} \& \mathrm{~W}$ Scientific (Agilent Technologies) was used to separate the methyl esters of the extracted fatty acids. Fatty acid concentrations were calculated in relation to the heptadecanoic acid methyl ester internal standard peak. The concentration of each phospholipid fatty acid was expressed as a concentration ( $\mu \mathrm{g} \cdot \mathrm{mL}^{-1}$ plasma).

Ferric-reducing antioxidant potential. Total plasma antioxidant potential was determined by the ferric-reducing antioxidant potential (FRAP) assay according to the methodology of Benzie and Strain (3). The basis of this assay is that water-soluble reducing agents (antioxidants) in the plasma will reduce ferric ions to ferrous ions, which then react with an added chromogen. Samples and standards were analyzed in duplicate and expressed as ascorbate equivalents on the basis of an ascorbate standard curve $(0-1000 \mu \mathrm{mol})$. Intraassay and interassay coefficients of variation were less than $5 \%$ and $7 \%$, respectively.

Oxygen radical absorption capacity (ORAC). The ORAC assay was a modification of the methodology of $\mathrm{Ou}$ et al. (24). Briefly, trolox standards were made from a trolox stock solution. A serial dilution of a $50-\mu \mathrm{m}$ trolox solution was made with a phosphate-buffered solution to produce $25,12.5$, and $6.25 \mu \mathrm{m}$ of trolox standards. A fluorescein (Sigma-Aldrich Chemicals, St. Louis, MO) working solution was made by pipetting $800 \mu \mathrm{L}$ of stock solution into $50 \mathrm{~mL}$ of phosphate buffer in a $50-\mathrm{mL}$ conical tube. Before use, the solution was incubated in the water bath at $37^{\circ} \mathrm{C}$ until thoroughly heated.

The AAPH solution was made by dissolving $0.108 \mathrm{~g}$ of AAPH (Wako Chemical, Richmond, VA) into $5 \mathrm{~mL}$ of

TABLE 1. Subject characteristics for baseline measures (values are presented as mean \pm SEM)

\begin{tabular}{|c|c|c|c|c|c|}
\hline Variable & $P(n=12)$ & VM $(n=12)$ & N3 $(n=11)$ & VN3 $(n=13)$ & $P$ Value \\
\hline Age $(y r)$ & 26.92 .8 & $22.2 \pm 0.9$ & 24.12 .4 & $25.2 \pm 2.1$ & 0.460 \\
\hline Body mass $(\mathrm{kg})$ & 67.43 .8 & $73 \pm 3.5$ & 68.93 .0 & $66.7 \pm 4.0$ & 0.761 \\
\hline $\mathrm{V}_{2 \text { peak }}\left(\mathrm{mL} \cdot \mathrm{kg}^{-1} \cdot \mathrm{min}^{-1}\right)$ & 58.83 .1 & $66.6 \pm 3.1$ & 62.93 .8 & $59.8 \pm 7.3$ & 0.408 \\
\hline Power $_{\max }(\mathrm{W})$ & 25118.5 & $309 \pm 14.6$ & 28413.9 & $281 \pm 16.8$ & 0.172 \\
\hline $\mathrm{HR}_{\max }$ (beats $\cdot \mathrm{min}^{-1}$ ) & 1852.8 & $187 \pm 2.2$ & 1883.0 & $187 \pm 3.0$ & 0.362 \\
\hline Body composition (\% fat) & 16.42 .7 & $14.5 \pm 1.8$ & 13.71 .8 & $14.8 \pm 1.7$ & 0.431 \\
\hline $\mathrm{BMI}\left(\mathrm{kg} \cdot \mathrm{m}^{-2}\right)$ & $21.1 \pm 1.2$ & $23.1 \pm 1.2$ & $21.7 \pm 0.7$ & $21.7 \pm 1.2$ & 0.671 \\
\hline Training $\left(\mathrm{km} \cdot \mathrm{wk}^{-1}\right)$ & $209 \pm 36.2$ & $240 \pm 31.4$ & $213 \pm 38.5$ & $264 \pm 38.5$ & 0.681 \\
\hline Training $\left(h \cdot d^{-1}\right)$ & $0.64 \pm 0.13$ & $1.14 \pm 0.22$ & $1.09 \pm 0.17$ & $1.02 \pm 0.19$ & 0.173 \\
\hline
\end{tabular}

P, placebo; VM, vitamin-mineral; N3, n-3 fatty acid; VN3, vitamin-mineral and $n-3$ fatty acid combination. 
TABLE 2. Performance measures for average of three exercise sessions (values are presented as mean $\pm \mathrm{SEM}$ ).

\begin{tabular}{|c|c|c|c|c|c|}
\hline Variable & $P(n=12)$ & VM $(n=12)$ & N3 $(n=11)$ & VN3 $(n=13)$ & $P$ Value \\
\hline Watts & $143 \pm 10.3$ & $175 \pm 8.5$ & $162 \pm 8.1$ & $160 \pm 9.9$ & 0.102 \\
\hline Power $\left(\% W_{\max }\right)$ & $56.8 \pm 0.2$ & $56.7 \pm 0.3$ & $56.8 \pm 0.2$ & $57.1 \pm 0.2$ & 0.822 \\
\hline Mean HR (beats'min ${ }^{-1}$ ) & $144 \pm 3.4$ & $137 \pm 1.8$ & $141 \pm 2.2$ & $139 \pm 2.6$ & 0.322 \\
\hline $\mathrm{HR}\left(\% \mathrm{HR}_{\max }\right)$ & $78.2 \pm 1.1$ & $73.4 \pm 1.0$ & $74.8 \pm 1.4$ & $74.6 \pm 1.9$ & 0.069 \\
\hline Mean $\mathrm{VO}_{2}\left(\mathrm{~mL} \cdot \mathrm{min}^{-1}\right)$ & $2589 \pm 180$ & $3025 \pm 153$ & $2725 \pm 170$ & $2846 \pm 193$ & 0.590 \\
\hline $\mathrm{VO}_{2}\left(\% \mathrm{VO}_{2 \text { peak }}\right)$ & $66.3 \pm 3.0$ & $63.2 \pm 2.2$ & $64.2 \pm 3.2$ & $65.1 \pm 3.7$ & 0.642 \\
\hline RPE (rating perceived exertion) & $13.0 \pm 0.3$ & $12.9 \pm 0.3$ & $13.4 \pm 0.4$ & $12.9 \pm 0.4$ & 0.136 \\
\hline
\end{tabular}

P, placebo; VM, vitamin-mineral; N3, n-3 fatty acid; VN3, vitamin-mineral and n-3 fatty acid combination.

incubated phosphate buffer immediately before the start of the assay. The microplate was prepared and loaded in a "forward-then-reverse" order to avoid possible positional errors. The edge wells were left empty or blank (phosphatebuffered working solution) to reduce the impact of "edge effect" on samples and standards particularly from temperature effects on the outside wells. Twenty microliters of sample, blank, and trolox standard solutions were pipetted into appropriate wells. Then, $200 \mu \mathrm{L}$ of fluorescein working solution was added to each well using an eight-channel micropipettor. A cover was placed on the microplate, and the plate and the contents were incubated at $37^{\circ} \mathrm{C}$ for at least $20 \mathrm{~min}$. Then, $20 \mu \mathrm{L}$ of AAPH working solution was added using an eight-channel micropipettor as quickly as possible. Final ORAC values $\left(\mu \mathrm{m} \cdot \mathrm{L}^{-1}\right.$ trolox) were made from the plate reader-derived area under the curve. Then, the final ORAC $_{\mathrm{FL}}$ values were calculated by using a quadratic regression equation $x=-b \pm \sqrt{ } b^{2}-4 a c+4 c y$. Excitation wavelength was $485 \mathrm{~nm}$, and emission wavelength was $520 \mathrm{~nm}$.

Statistical analysis. Data are expressed as mean \pm SEM. All data were screened for outliers with outliers designated as being outside of the mean \pm 2.5 SD. Data in Tables 1 and 2 were compared between groups using Student's $t$-tests. All other data (Figs. 1-5) were analyzed using a 4 (groups) $\times 3$ (time points) repeated-measures ANOVA to determine overall main effects. To account for multiple comparisons between treatments, a Bonferroni adjustment was made and the significant $P$ value established as $P \leq 0.017$ and the data compared using one-way ANOVA. These data analysis allowed group comparisons for two different effects: $6 \mathrm{wk}$ of chronic supplementation and acute postexercise changes.

\section{RESULTS}

Descriptives. Subject baseline characteristics are summarized in Table 1. Outcome data for male and female cyclists did not differ significantly and are presented together for each group. During the 3 -h cycling bouts, temperature averaged $22.4^{\circ} \mathrm{C} \pm 0.06^{\circ} \mathrm{C}$ and relative humidity $35.8 \% \pm 1.6 \%$. No significant differences were found among groups for basic physical characteristics (Table 1). Subject performance characteristics were averaged over the three exercise sessions and are presented in Table 2. There was no effect of treatment group on any exercise related performance variable. Plasma volume change did not differ between groups postexercise on the third day and averaged less than $2 \%$ because of ingestion of $0.5-1.0 \mathrm{~L}$ of water per hour of exercise (data not shown).

Oxidative, n-3fa, and antioxidant variables. EPA and DHA supplementation resulted in drastic increases from baseline to preexercise. DHA main effects were treatment $(P=0.068)$, time $(P=0.004)$, and interaction $(P=0.006)$.

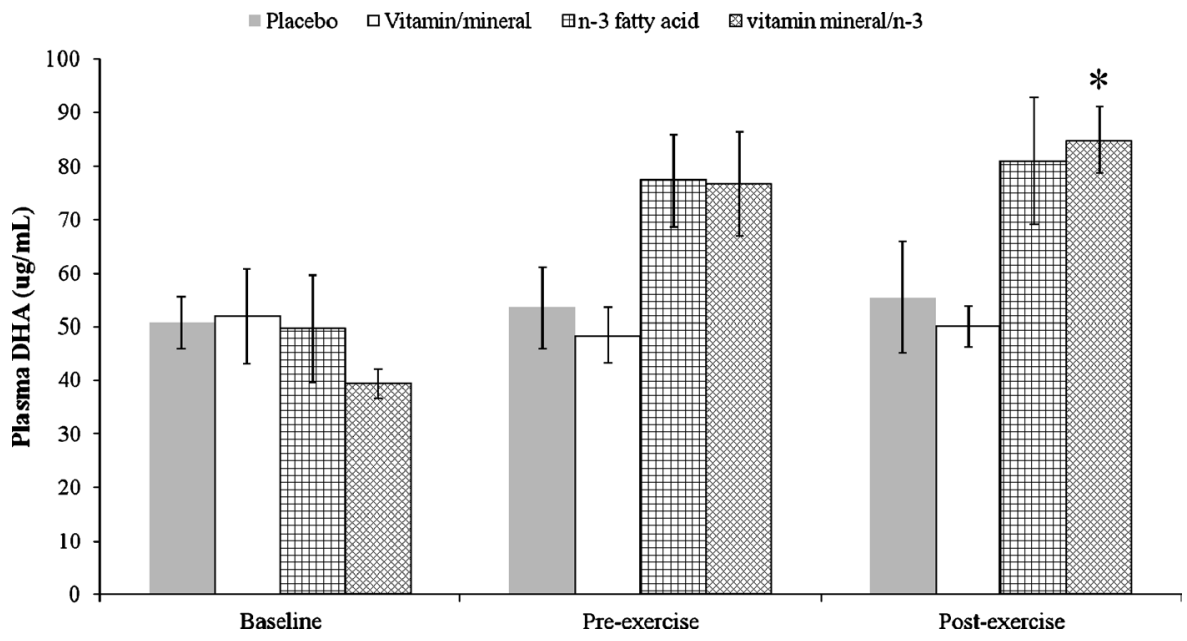

FIGURE 1-Plasma DHA values over time among four treatments. Treatment effect $(P=0.184)$, time effect $(P=0.002)$, and treatment $\times$ time interaction $(P=0.006) . * P \leq 0.0125$ after Bonferroni correction versus placebo value at baseline $(n=8)$. Values are presented as mean \pm SEM. 


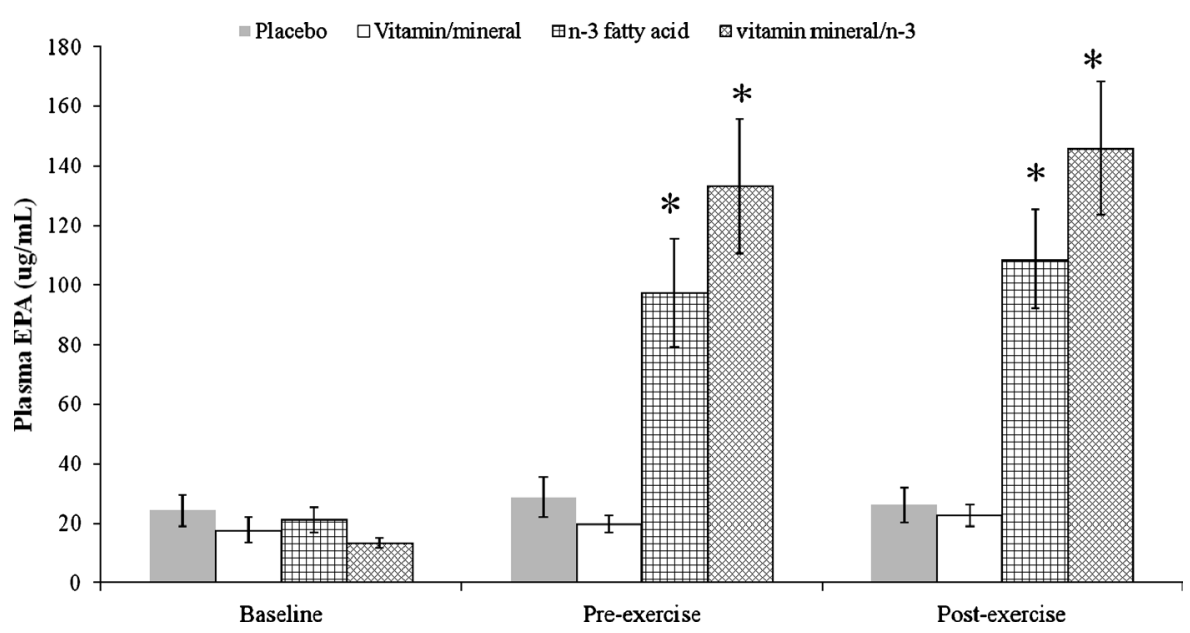

FIGURE 2-Plasma EPA values over time among four treatments. Treatment effect $(P=0.001)$, time effect $(P=0.001)$, and treatment $\times$ time interaction $(P=0.001) . * P \leq 0.0125$ after Bonferroni correction versus placebo value at baseline $(n=9)$. Values are presented as mean \pm SEM.

Plasma DHA increased 55.8\% in N3 and 95.3\% in VN3 from baseline to preexercise, respectively (Fig. 1). EPA exhibited significant treatment $(P \leq 0.001)$, time $(P \leq 0.001)$, and interaction effects $(P \leq 0.001)$. Plasma EPA increased $61.5 \%$ in $\mathrm{N} 3$ and $778 \%$ in $\mathrm{VN} 3$ from baseline to preexercise (Fig. 2). The change in $\mathrm{F}_{2}$-isoprostanes produced significant overall treatment effects $(P=0.014)$ and time effects $(P=0.001)$. Interestingly, $\mathrm{F}_{2}$-isoprostanes increased approximately $53 \%$ from baseline to immediately postexercise in N3. This change was significantly higher versus the change observed in $P(P \leq 0.01)$. VM and VN3 groups were not different from $P$ (Fig. 3). When specific comparisons of the pattern of change in $\mathrm{F}_{2}$-isoprostanes from baseline to postexercise were made between treatments, $\mathrm{F}_{2}$-isoprostanes in N3 were higher versus $\mathrm{P}(P=0.020)$, higher versus VM $(P=0.008)$, and higher versus VN3 $(P=0.05)$ (Fig. 3).

ORAC declined similarly and significantly among all groups $(P \leq 0.001)$ from preexercise, but the pattern of change was not different between groups $(P=0.217)$. The exercise-induced decline was greatest in $\mathrm{P}(37 \%)$ versus N3 (23\%), VM (23\%), and VN 3 (21\%) (Fig. 4). FRAP plasma antioxidant capacity values were higher after exercise among all groups. However, only the vitamin and mineral containing groups exhibited significant differences from baseline $(P \leq 0.017)$ after Bonferroni correction. Overall effects for FRAP values were treatment $(P=0.300$, time $(P=0.003)$, and interaction $(P=0.045)$. FRAP increased $26 \%$ immediately postexercise in VN $3,19 \%$ in VM, 5.9\% in N3, and $9.5 \%$ in P (Fig. 5).

\section{DISCUSSION}

One of the most important outcomes of this investigation was that consumption of a supplement containing $2000 \mathrm{mg}$ of EPA and $400 \mathrm{mg}$ of DHA daily for 6 wk and during $3 \mathrm{~d}$ of intense exercise resulted in a significantly larger increase (53\% increase from preexercise) in $\mathrm{F}_{2}$-isoprostanes compared

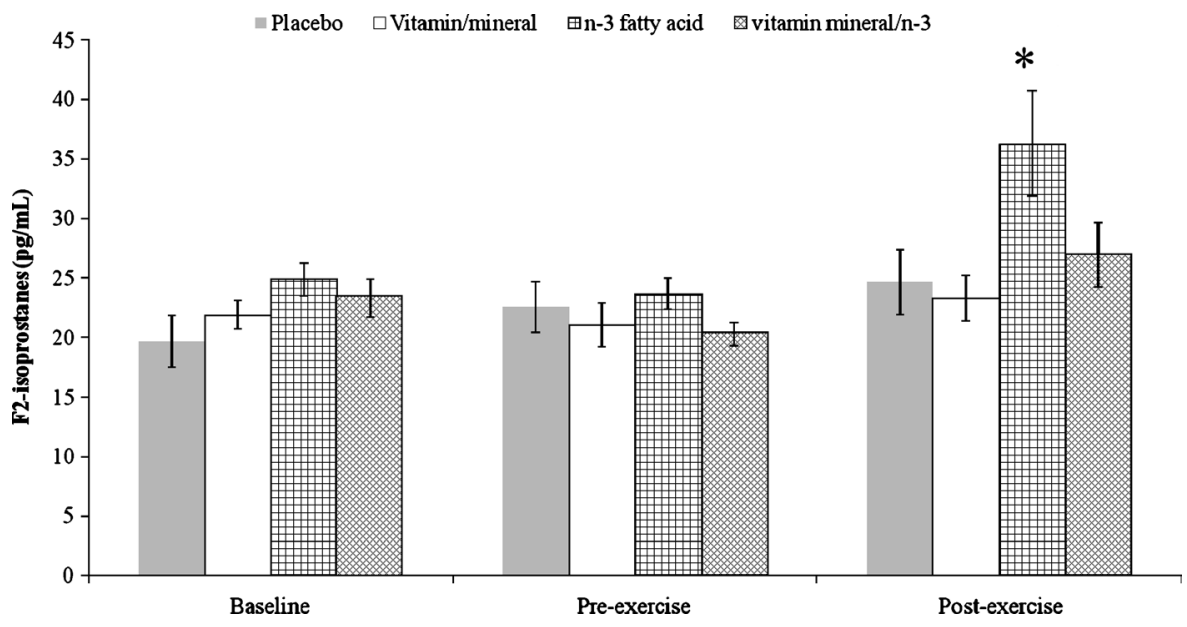

FIGURE 3- $F_{2}$-isoprostane values over time among four treatments. Treatment effect $(P=0.014)$, time effect $(P=0.002)$, and treatment $\times$ time interaction $(P=0.224) . * P \leq 0.0125$ after Bonferroni correction versus placebo value at baseline $(n=12)$. Values are presented as mean \pm SEM. 


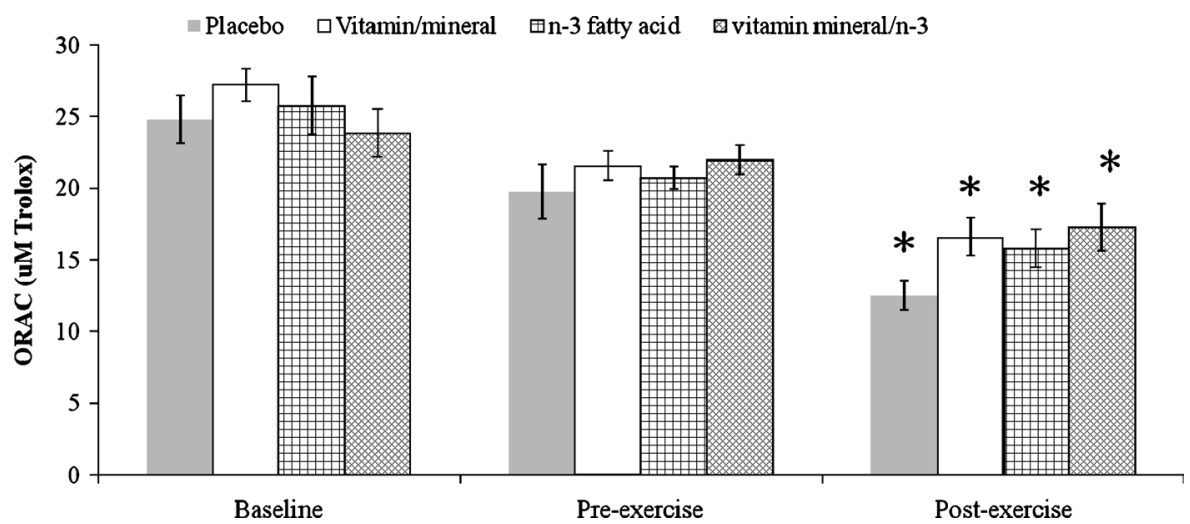

FIGURE 4-Plasma ORAC values over time among four treatments. Treatment effect $(P=0.187)$, time effect $(P=0.001)$, and treatment $\times$ time interaction $(P=0.381)$. ${ }^{*} P \leq \mathbf{0 . 0 1 2 5}$ after Bonferroni correction versus placebo value at baseline $(n=10)$. Values are presented as mean \pm SEM.

with placebo and other antioxidant and n-3fa combinations after exhaustive exercise. This is of some importance given that $\mathrm{F}_{2}$-isoprostanes are bioactive and elevated in obesity and various other disease states (20). This outcome supports previous concerns that an increased ingestion of polyunsaturated fatty acids, whether n-3fa or n-6fa , might lead to increased lipid peroxidation (19). As further evidence that $n-3$ fa enhanced lipid peroxidation in our study, $\mathrm{F}_{2}$-isoprostanes were only increased by $10 \%$ or less within the treatment groups that did not contain n-3fa. These smaller changes in $\mathrm{F}_{2}$-isoprostanes are similar to other studies we have conducted, indicating that longer duration exercise (exceeding $1 \mathrm{~h}$ ) does not increase $\mathrm{F}_{2}$-isoprostanes to the degree of shorter intense exercise $(17,23)$.

Several interesting points emerge from our investigation. Although it is clear that increased n-3fa ingestion can result in increased lipid peroxidation $(11,29)$, the $n-3$ fa peroxidation products do not directly result in production of $\mathrm{F}_{2-}$ isoprostanes. Instead, the main products are $\mathrm{F}_{3}$-isoprostanes (from EPA) and $\mathrm{F}_{4}$-isoprostanes (from DHA) (30) as well as the aldehyde fragment 4-hydroxy-2E-hexenal (11). These compounds have been found to be increased in tissues submitted to oxidative stress $(11,30)$. As $\mathrm{F}_{2}$-isoprostanes derive solely from the oxidation of arachidonate (20), this implies that downstream n-3fa peroxidation products or some other mechanism increases $\mathrm{F}_{2}$-isoprostanes indirectly. In support of this hypothesis, Catala (6) states that lipid hydroperoxides can participate in chain reactions that increase damage to other lipid biomolecules. Most hydroperoxides are reduced to corresponding alcohols by glutathione peroxidases but some degrade into other compounds, including hydroxy-alkenals. The best known of these are 4-hydroxy2-nonenal (4-HNE) and 4-hydroxy-2E-hexenal, which derive from lipid peroxidation of $n-6 f a$ and $n-3 f a$, respectively. These aldehydes are relatively stable and can diffuse within or escape from the cell and attack other biomolecules distant from the original event. These aldehydes exhibit great reactivity with proteins, DNA, and membrane bound phospholipids. Arachidonic acid is prominent in membrane phospholipids that would oxidize directly to produce $\mathrm{F}_{2}$-isoprostanes (20).

Another observation is that n-3fa supplementation typically reduces baseline concentrations of $F_{2}$-isoprostanes (19). Nalsen et al. (22) found that supplementation with a total of $3.6 \mathrm{~g} \mathrm{n}-3 \mathrm{fa}$ (2.4g EPA and DHA combined) per day significantly reduced $\mathrm{F}_{2}$-isoprostane concentrations in nonexercising healthy humans after 3 months versus placebo. Mori et al. (19) provided daily fish meals or EPA and DHA

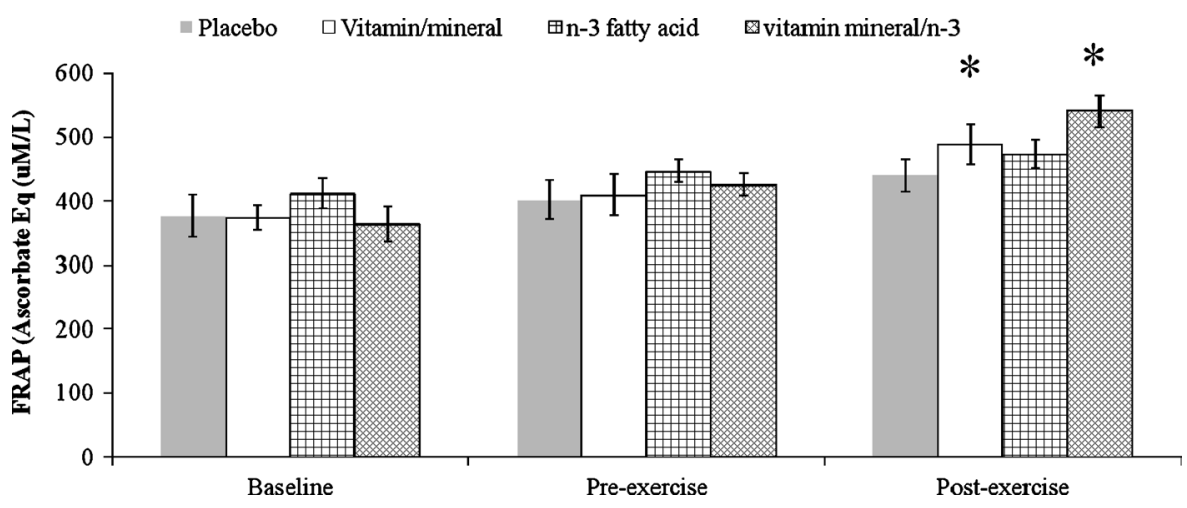

FIGURE 5-FRAP values over time among four treatments. Treatment effect $(P=0.409)$, time effect $(P=0.002)$, and treatment $\times$ time interaction $(P=0.035) . * P \leq 0.0125$ after Bonferroni correction versus placebo value at baseline $(n=12)$. Values are presented as mean \pm SEM. 
and found that urinary $\mathrm{F}_{2}$-isoprostanes were significantly reduced by $20 \%-27 \%$ in both trials. We did not observe this decrease from baseline with $6 \mathrm{wk}$ of supplementation. An interesting difference is that our entire subject population were athletes engaged in routine heavy endurance exercise versus those of Mori et al. (19) and Nalsen et al. (22).

Because the concentration of $\mathrm{F}_{2}$-isoprostanes was so drastically increased postexercise versus other treatments, we hypothesize that consumption of high amounts of $n-3$ fa (14) does not increase peroxidation until a state of high oxidative stress is initiated. Excessive radical formation and trauma during exercise leads to an inflammatory state that may propagate overall lipid peroxidation and increase $\mathrm{F}_{2}$-isoprostanes as addressed earlier. Because n-3fa are apparently susceptible to oxidation during exercise, it becomes important whether the chain reaction of lipid peroxidation can be interrupted by increased dietary antioxidants and whether increased concentrations of $n-3 f a$ in the cellular membrane increase antioxidant requirements. $\mathrm{F}_{2}$-isoprostanes were increased $53 \%$ by $\mathrm{n}-3$ fatty acids alone but only $32.8 \%$ by the same amount of $n-3$ fa coupled with large-dose antioxidant vitamins. Interestingly, this suggests that coingestion of antioxidants with the $n-3$ fa offered some protection from oxidation. Although using a less sensitive indicator of lipid peroxidation, Allard et al. (1) examined supplementation with a total of $6.26 \mathrm{~g}$ of EPA and DHA n-3fa daily for 6 wk in 80 healthy men alone or in combination with alpha-tocopherol (900 IU of vitamin E) versus placebo on lipid peroxidation. Supplementation with $\mathrm{n}-3 \mathrm{fa}$ significantly increased plasma MDA and lipid peroxides. However, consumption of vitamin $\mathrm{E}$ concurrent with the n-3fa did not reduce the increase in lipid peroxidation. In contrast, we used combinations of antioxidants added to the n-3fa supplement, which may have been more effective and accounted for the attenuation in $\mathrm{F}_{2}$-isoprostanes observed in the n-3 and antioxidant combination group (16).

FRAP plasma antioxidant capacity values were higher after exercise among all groups. However, only the vitamin and mineral containing groups exhibited significant differences from baseline. The FRAP assay has been found to respond strongly to the presence of water-soluble antioxidants such as urate and vitamin C (3). Interestingly, vitamin and mineral supplementation did not significantly increase ORAC value compared with placebo. Plasma ORAC values declined significantly and similarly between treatments after exercise, suggesting depletion of an antioxidant or multiple antioxidants, which was not prevented by antioxidant supplementation. The uniqueness of the ORAC assay is that total antioxidant capacity of a sample is estimated by taking an oxidation reaction to completion. ORAC measures all of the nonprotein antioxidants, which include alpha-tocopherol, glutathione, vitamin $\mathrm{C}$, beta-carotene, uric acid, and bilirubin. In this regard, ORAC constitutes more of a comprehensive antioxidant measurement encompassing multiple antioxidants.
Whether strenuous exercise greatly increases the need for additional antioxidant vitamin and minerals in the diet or through supplementation is not clear. Interestingly, the opposite may be true because Gomez-Cabrera et al. (10) and Ristow et al. (25) found that supplementation with antioxidant vitamins actually reduced beneficial adaptations from exercise and therefore might be contraindicated. Nonetheless, as previously mentioned, we observed higher FRAP values postexercise in the groups receiving vitamin and minerals, but this did not affect $\mathrm{F}_{2}$-isoprostanes. However, Mastaloudis et al. (16) found that 6 wk of supplementation with $1000 \mathrm{mg}$ vitamin C and $300 \mathrm{mg}$ RRR-alpha-tocopheryl acetate prevented exercise-induced $\mathrm{F}_{2}$-isoprostanes but had no effect on inflammatory markers after a $50-\mathrm{km}$ ultramarathon. McAnulty et al. (17) examined the effect of 2 months of vitamin E supplementation (800 IU.d of alpha-tocopherol) in highly trained athletes during exhaustive exercise on plasma antioxidant potential and $\mathrm{F}_{2}$-isoprostanes. Conversely, this study found that prolonged large doses of alpha-tocopherol supplementation did increase plasma antioxidant potential postexercise, but lipid peroxidation was actually higher in the supplemented group versus placebo. Nieman et al. (23) evaluated the influence of vitamin $C$ supplementation (1500 $\mathrm{mg} \cdot \mathrm{d}^{-1}$ ) during the $7-\mathrm{d}$ period before the race and on race day compared with placebo on $\mathrm{F}_{2}$-isoprostanes in runners competing in an ultramarathon race. Despite higher plasma vitamin $\mathrm{C}$ concentrations, no significant effects were measured for lipid hydroperoxides or $\mathrm{F}_{2}$-isoprostanes. Evidently, multiple factors such as timing, type and concentration of antioxidants, and mode of exercise influence the outcomes associated with antioxidant supplementation.

In conclusion, despite the beneficial effects typically associated with n-3fa ingestion, we observe for the first time evidence that supplementation with $\mathrm{n}-3 \mathrm{fa}$ is associated with significantly increased $\mathrm{F}_{2}$-isoprostanes in response to heavy cycling exercise. Supplementation with antioxidant vitamin combinations and minerals did not significantly influence $\mathrm{F}_{2}$-isoprostane formation versus placebo. Furthermore, $\mathrm{n}-3 \mathrm{fa}$ supplementation did not influence any measure of plasma antioxidant capacity, but a vitamin and mineral supplementation was effective at increasing FRAP values. On the basis of our results, further assessment is clearly needed to examine whether the possible benefits of $n-3$ fa supplementation would outweigh the potential risks of increased lipid peroxidation in athletes who are engaged in heavy exercise. Furthermore, because this study was conducted in a young fit population, future studies should include aging or older adults, whom often are prescribed exercise and fish oils to promote cardiovascular health because aging compromises antioxidant defenses (13).

This project was funded by the Cooper Aerobics Center, Dallas, Texas.

The results of this study do not constitute endorsement by the American College of Sports Medicine. 


\section{REFERENCES}

1. Allard JP, Kurian R, Aghdassi E, Muggli R, Royall D. Lipid peroxidation during n-3 fatty acid and vitamin $\mathrm{E}$ supplementation in humans. Lipids. 1997;32:535-41.

2. Basarici I, Altekin RE, Demir I, Yilmaz H. Urinary 8-isoprostane levels can indicate the presence, severity and extent of angiographic coronary artery disease. Acta Cardiol. 2008;63:415-22.

3. Benzie IFF, Strain JJ. The ferric reducing ability of plasma (FRAP) as a measure of "antioxidant power": the FRAP assay. Anal Biochem. 1996;239:70-6.

4. Bjerregaard P, Pedersen HS, Mulvad G. The associations of a marine diet with plasma lipids, blood glucose, blood pressure and obesity among the inuit in Greenland. Eur J Clin Nutr. 2000; 54:732-7.

5. Blair SN, Kohl HW III, Paffenbarger RS Jr, Clark DG, Cooper KH, Gibbons LW. Physical fitness and all-cause mortality. A prospective study of healthy men and women. JAMA. 1989; 262:2395-401.

6. Catala A. Lipid peroxidation of membrane phospholipids generates hydroxy-alkenals and oxidized phospholipids active in physiological and/or pathological conditions. Chem Phys Lipids. 2009; 157:1-11.

7. Chan DW, Liu VW, Tsao GS, et al. Loss of MKP3 mediated by oxidative stress enhances tumorigenicity and chemoresistance of ovarian cancer cells. Carcinogenesis. 2008;29:1742-50.

8. Das UN. Beneficial effect(s) of n-3 fatty acids in cardiovascular diseases: but, why and how? Prostaglandins Leukot Essent Fatty Acids. 2000;63:351-62.

9. Dill DB, Costill DL. Calculation of percentage changes in volumes of blood, plasma, and red cells in dehydration. $J$ Appl Physiol. 1974;37:247-8.

10. Gomez-Cabrera MC, Martinez A, Santangelo G, Pallardo FV, Sastre J, Vina J. Oxidative stress in marathon runners: interest of antioxidant supplementation. Br J Nutr. 2006;96(1 suppl): S31-3.

11. Guichardant M, Bacot S, Moliere P, Lagarde M. Hydroxyalkenals from the peroxidation of n-3 and n-6 fatty acids and urinary metabolites. Prostaglandins Leukot Essent Fatty Acids. 2006;75:179-82.

12. Ji LL, Gomez-Cabrera MC, Vina J. Exercise and hormesis: activation of cellular antioxidant signaling pathway. Ann $N Y$ Acad Sci. 2006;1067:425-35.

13. Ji LL, Leeuwenburgh $\mathrm{C}$, Leichtweis $\mathrm{S}$, et al. Oxidative stress and aging. Role of exercise and its influences on antioxidant systems. Ann N Y Acad Sci. 1998;854:102-17.

14. Kris-Etherton PM, Harris WS, Appel LJ. Omega-3 fatty acids and cardiovascular disease: new recommendations from the American Heart Association. Arterioscler Thromb Vasc Biol. 2003;23:151-2.
15. Massaro M, Scoditti E, Carluccio MA, De CR. Basic mechanisms behind the effects of $\mathrm{n}-3$ fatty acids on cardiovascular disease Prostaglandins Leukot Essent Fatty Acids. 2008;79:109-15.

16. Mastaloudis A, Morrow JD, Hopkins DW, Devaraj S, Traber MG. Antioxidant supplementation prevents exercise-induced lipid peroxidation, but not inflammation, in ultramarathon runners. Free Radic Biol Med. 2004;36:1329-41.

17. McAnulty SR, McAnulty LS, Nieman DC, et al. Effect of alphatocopherol supplementation on plasma homocysteine and oxidative stress in highly trained athletes before and after exhaustive exercise. J Nutr Biochem. 2005;16:530-7.

18. Mori TA. Effect of fish and fish oil-derived omega-3 fatty acids on lipid oxidation. Redox Rep. 2004;9:193-7.

19. Mori TA, Puddey IB, Burke V, et al. Effect of omega 3 fatty acids on oxidative stress in humans: GC-MS measurement of urinary $\mathrm{F}_{2}$-isoprostane excretion. Redox Rep. 2000;5:45-6.

20. Morrow JD, Roberts LJ. The isoprostanes: unique bioactive products of lipid peroxidation. Prog Lipid Res. 1997;36:1-21

21. Morrow JD, Roberts LJ. Mass spectrometric quantification of $\mathrm{F}_{2}$-isoprostanes in biological fluids and tissues as measure of oxidant stress. Methods Enzymol. 1999;300:3-12.

22. Nalsen C, Vessby B, Berglund L, et al. Dietary (n-3) fatty acids reduce plasma $\mathrm{F}_{2}$-isoprostanes but not prostaglandin $\mathrm{F} 2$ alpha in healthy humans1. J Nutr. 2006;136:1222-8.

23. Nieman DC, Henson DA, McAnulty SR, et al. Influence of vitamin $\mathrm{C}$ supplementation on oxidative and immune changes after an ultramarathon. $J$ Appl Physiol. 2002;92:1970-7.

24. Ou B, Hampsch-Woodill M, Prior RL. Development and validation of an improved oxygen radical absorbance capacity assay using fluorescein as the fluorescent probe44. J Agric Food Chem. 2001;49:4619-26.

25. Ristow M, Zarse K, Oberbach A, et al. Antioxidants prevent health-promoting effects of physical exercise in humans. Proc Natl Acad Sci U S A. 2009;106:8665-70.

26. Samieri C, Feart C, Letenneur L, et al. Low plasma eicosapentaenoic acid and depressive symptomatology are independent predictors of dementia risk. Am J Clin Nutr. 2008;88:714-21.

27. Simopoulos AP. The importance of the ratio of omega-6/omega-3 essential fatty acids. Biomed Pharmacother. 2002;56:365-79.

28. Simopoulos AP. Omega-3 fatty acids and athletics. Curr Sports Med Rep. 2007;6:230-6.

29. Valencak TG, Ruf T. N-3 polyunsaturated fatty acids impair lifespan but have no role for metabolism. Aging Cell. 2007;6:15-25.

30. Yin H, Liu W, Goleniewska K, Porter NA, Morrow JD, Peebles RS Jr. Dietary supplementation of omega-3 fatty acid-containing fish oil suppresses $\mathrm{F}_{2}$-isoprostanes but enhances inflammatory cytokine response in a mouse model of ovalbumin-induced allergic lung inflammation. Free Radic Biol Med. 2009;47:622-8. 